\title{
The Spectrum of Large Adrenal Masses: A Case Series
}

Jo Cooke-Barber, MD, ${ }^{1}$ Sarah E. Hatef, BS, MPH, ${ }^{2}$ Analise McGreal, BS, ${ }^{3}$

Andrew Schwemmer, MD, ${ }^{1}$ Christopher Senkowski, MD ${ }^{1,3}$

\section{Abstract}

\section{Background}

Adrenocortical carcinoma (ACC) is a rare malignancy that is challenging to diagnose and has important implications for surgeons who approach this disease. Despite its rarity, it must always be in the differential diagnosis when investigating and treating large adrenal masses. We aim to demonstrate the complexities of this disease through a review of five recent patients at a single tertiary care center.

Author affiliations are listed at the end of this article.

Correspondence to:

Analise McGreal

Mercer School of Medicine 1250 East 66th Street Savannah, GA 31404 (Analise.Elizabeth.Mcgreal@live.mercer.edu)

\section{Methods}

A series of five patients are described, each of whom presented to a single institution as referrals for "large adrenal mass" in the past sixteen months. Their pre-operative diagnosis, radiographic findings, the operative approach and the pathology results were examined.

\section{Results}

The first patient had a $12 \mathrm{~cm}$ high grade adrenocortical carcinoma. The second patient had pathology consistent with a $9 \mathrm{~cm}$, high grade $A C C$. The third had a liposarcoma. The fourth patient had a myelolipoma. The fifth was diagnosed with a benign process.

\section{Conclusion}

Although adrenocortical carcinoma is an uncommon cancer, it has significant implications for the patient's prognosis and ultimately, their treatment algorithm. Therefore, when evaluating large adrenal masses, surgeons must remain vigilant of the possibility of adrenocortical carcinoma.

\section{Keywords}

adrenal cortex neoplasms; adrenocortical carcinoma; pheochromocytoma; adrenal glands; neoplasm metastasis; carcinoma, diagnosis; carcinoma, pathology

\section{Introduction}

Adrenocortical carcinoma is a rare form of cancer accounting for about $0.02 \%$ of all carcinomas, ${ }^{1}$ with just two cases per million people in the world and 200 new cases per year in the United States. ${ }^{2}$ Nonetheless, when investigating a large adrenal mass, especially greater than ten centimeters, adrenocortical carcinoma must be high on the differential diagnosis.

Adrenocortical cancer (ACC) affects women more than men and has a bimodal age distribution with peaks in the first and fourth decades of life. ${ }^{2,3}$ It has no geographic pattern and no confirmed etiology. ACC has been found to be associated with several genetic factors including insulin-like growth factor overexpression, constitutive activation of the Wnt-b-catenin signaling pathway, as well as TP53 tumor suppressor mutations. ${ }^{4}$ Additionally, studies have shown a germline mutation in TP53 in certain Brazilian populations which correlates with an ACC prevalence in Brazilian children 10-15 times greater than that of the world pediatric population. ${ }^{5}$ This association, along with the strong association between ACC and Li-Fraumeni syndrome, offer further evidence for TP53's role in adrenocortical carcinoma. In addition to www.hcahealthcarejournal.com

(C) 2020 HCA Physician Services, Inc. d/b/a Emerald Medical Education
HCA Healthcare Journal of Medicine 
Li-Fraumeni syndrome, higher rates of ACC are found in those with Beckwith-Widemann syndrome and Carney complex, implicating genes IGF2 and PRKAR1A in the pathogenesis. ${ }^{4}$

Diagnosis and determination of prognosis are of utmost importance in ACC, since prompt surgical intervention and complete surgical resection are the only known curative treatments for the disease (5-year disease free survival), lowering recurrence rates below $30 \% .{ }^{6}$ Surgical resection is the mainstay of treatment, followed by adjuvant chemotherapy with mitotane and radiation. Patient prognosis can be predicted with relative accuracy using the European Network for the Study of Adrenal Tumors (ENSAT) Staging Score. (Table 1) The ENSAT Staging Score not only assists clinicians in giving prognostic information to their patients, but aids in choosing a therapeutic approach as well. ${ }^{6}$ A retrospective review of 330 patients at MD Anderson Cancer Center in Texas found that the factors associated with local recurrence were positive surgical margins and advanced disease stage. In addition, the factors associated with poor survival were functioning tumors, R1/R2 surgical resections, stage III/IV at diagnosis and associated venous thromboembolism. ${ }^{7}$

Table 1. ENSAT Staging Score

\begin{tabular}{l|l} 
Score & $\begin{array}{l}\text { Components of Staging } \\
\text { Score }\end{array}$ \\
\hline I & T1, NO, MO \\
\hline II & T2, NO, MO \\
\hline III & T3-T4, N1 \\
\hline IV & T1-T4, NO-N1, M1
\end{tabular}

T1, tumor $\leq 5 \mathrm{~cm}$

T2, tumor $>5 \mathrm{~cm}$

T3, histologically proven tumor invasion of surrounding tissue

T4, tumor invasion of adjacent organs or venous tumor thrombus in vena cava or renal vein. (Venous tumor thrombus is a criterion for poorer prognosis in the ENSAT classification.)

NO, negative lymph nodes

$\mathrm{N} 1$, positive lymph nodes

$\mathrm{MO}$, absence of distant metastases

M1, presence of distant metastases

Despite the importance of a timely diagnosis, the ability to diagnose ACC pre-surgically remains limited. ACC commonly presents with steroid hormone excess and rapidly progress- ing Cushing's syndrome with or without virilization. ${ }^{3}$ Other clinical presentation patterns in women include androgen-secreting tumors which lead to hirsutism, virilization, dysphonia, male-patterned baldness and amenorrhea. In males, estrogen-secreting tumors cause testicular atrophy, which can lead to gynecomastia and baldness. Aldosterone-secreting ACC can cause hypertension and hypokalemia. ${ }^{1}$

Surprisingly, high levels of steroid precursors can also be found in hormonally inactive ACC possibly simply due to the size of these tumors. These hormonally inactive tumors commonly present with abdominal mass effect involving the kidney causing hematuria and dysuria. Abdominal pain, nausea, vomiting or back pain may also be present. ACC can also invade vasculature such as the left renal vein or the inferior vena cava leading to embolism, thrombosis or occlusion.

Although ACC can present in several ways, it is often asymptomatic and studies have demonstrated that up to $15 \%$ are discovered incidentally. ${ }^{3} \mathrm{Li}$ et al. studied 1,941 patients with adrenal incidentaloma (Al) and found that only $1.62 \%$ of them were diagnosed with ACC. ${ }^{8}$ For this reason, the disease is often discovered in advanced stages. Thus, $20-30 \%$ of patients with adrenocortical carcinoma already have distant metastases at the time of diagnosis and the 5-year survival for this disease is low, ranging between $16-40 \% .{ }^{9}$ Improving the ability to diagnose ACC preoperatively could potentially increase survival. The purpose of this case series is to identify common issues in diagnosing adrenocortical carcinoma and to offer insight into choosing the best surgical approach.

The diagnosis of $A C C$ relies on careful investigation of clinical, biological and imaging features before surgery and pathological examination. The ENSAT Staging Score suggests a preoperative hormonal workup for suspected ACC. In particular, the assessment of basal cortisol, ACTH, dehydroepiandrostenedione sulfate, 17-hydroxyprogesterone, testosterone, androstenedione and estradiol, as well as a dexamethasone suppression test and urinary free cortisol are recommended. ${ }^{3}$

The risk for $A C C$ increases with tumor size, with the index of suspicion increasing for tu- 
mors > $4 \mathrm{~cm}$ (sensitivity, 97\%; specificity, 52\%) and > $6 \mathrm{~cm}$ (sensitivity, 91\%; specificity, 80\%). ${ }^{10}$ An abdominal computed tomography (CT) scan is mandatory in suspicion of ACC, studies have established a threshold of $\leq 10$ Hounsfield Unit (HU) in unenhanced CT for the diagnosis of benign lesions. If the basal density is $>10$ $\mathrm{HU}$, a contrast media washout will be helpful in differentiating a benign lesion from ACC; an absolute washout $>50 \%$ suggests a benign lesion.11-14 If CT imaging cannot adequately characterize the adrenal mass, three major characteristics of an MRI will be helpful in diagnosing ACC: the presence of isointense to hypointense signal on T1-weighted images, a hyperintense signal on T2-weighted images and a heterogeneous signal drop on chemical shift. ${ }^{15}$

Although a percutaneous adrenal biopsy was widely used in the past, with the advances in imaging modalities and the associated complications with biopsy, it is less frequently used at present. ${ }^{16}$ Moreover, it is difficult to differentiate an adrenal adenoma from ACC with a fine needle aspiration (FNA) biopsy..$^{17}$ The clinical indications for a needle biopsy are the presence of an adrenal mass in an oncologic patient for staging or characterization of a primary lesion when the differentiation of benign from malignant lesions using imaging findings is not resolved. ${ }^{17}$

\section{Methods}

Five patients are presented from Memorial Health University Medical Center. All referrals for "large adrenal lesion" over a 16-month period were selected for inclusion in this case series. All patients received pre-operative imag- ing, which is reviewed. The surgical approaches for each case are discussed. The primary outcome was presence or absence of pathology consistent with adrenocortical carcinoma.

\section{Results \\ Case 1}

A 32-year-old female with no significant past medical history presented to her obstetrician 9 months post-partum with complaints of oligomenorrhea, hirsutism, and maculopapular rash. Laboratory workup revealed serum cortisol of $28 \mathrm{mcg} / \mathrm{dL}$ (ref: $10-20 \mathrm{mcg} / \mathrm{dL}$ ), ACTH < $1.1 \mathrm{pg} /$ $\mathrm{mL}$ (ref: $10-60 \mathrm{pg} / \mathrm{mL}$ ) and salivary cortisol of $2.22 \mathrm{mcg} / \mathrm{dL}$ (ref: 0.09-0.6 mcg/dL). Serum androgens were elevated, including DHEAS at 669 mcg/dL (ref: 17-286 mcg/dL), 17-OH progesterone at $84 \mathrm{ng} / \mathrm{dL}$ (ref: < $200 \mathrm{ng} / \mathrm{dL}$ ), total testosterone at $78 \mathrm{ng} / \mathrm{dL}$ (ref: $8-48 \mathrm{ng} / \mathrm{dL}$ ) and free testosterone at $16.3 \mathrm{pg} / \mathrm{mL}$ (ref: 0-4.2 pg/ $\mathrm{mL}$ ), suggestive of an adrenal etiology. Computed tomography (Figure 1) demonstrated a $12.2 \times 7.1 \times 7.5 \mathrm{~cm}$ heterogeneously enhancing mass containing scattered calcifications in the left adrenal gland with displacement of left kidney, pancreatic tail, spleen and adjacent bowel.

The patient was referred to the surgical oncology clinic to discuss treatment options for a functional left adrenal mass. An open left adrenalectomy via abdominal approach with potential en bloc resection of spleen and distal pancreas was offered, given significant malignant potential of the mass. The patient underwent a radical open left adrenalectomy, including partial diaphragm resection to ensure adequate margins and lymphadenectomy. During the lymphadenectomy, bulky perihilar lymph

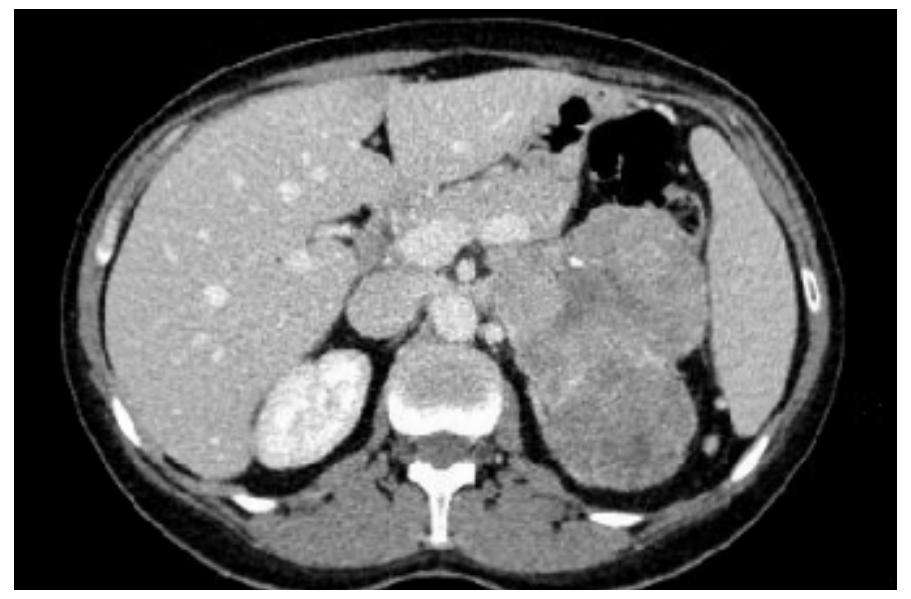

Figure 1. CT abdomen/pelvis revealed a left adrenal mass. 
nodes required resection of renal vessels and necessitated a left nephrectomy. Pathology revealed a high-grade adrenocortical carcinoma with positive nodes. The kidney was uninvolved and diaphragm margins were negative. Resection of the spleen and distal pancreas was not required. At time of resection, the tumor was Stage III (T4, N1, MO).

Surveillance imaging demonstrated hepatic metastases 3 months after her surgery and an area concerning for local recurrence along the left retroperitoneum. Cytoreductive ablation was performed for the hepatic lesions. Additionally, she was referred to medical oncology and underwent adjuvant chemotherapy with mitotane, etoposide, doxorubicin and cisplatin, which yielded minimal results. Second line treatment was subsequently undertaken with four cycles of pembrolizumab. Patient expired from disease at 8 months from resection.

\section{Case 2}

A 72-year-old female presented to her primary care physician for complaints of vague abdominal pain, weight loss, headaches, sore throat, voice changes, leg swelling, back pain, skin changes, nausea and diarrhea. Esophagogastroduodenoscopy and colonoscopy revealed esophageal candidiasis and gastritis. The patient's past medical history was pertinent for thyroid disease, HTN, anemia, arthritis, glaucoma, uterine and breast cancer, and tobacco use. The patient's past surgical history was pertinent for a hysterectomy, hernia repair, laparoscopic gastric banding and left mastectomy. Her family history was positive for cancer, diabetes, heart disease and hypertension. The patient's physical exam demonstrated tender- ness in the left upper quadrant. Outside hospital non-contrasted CT imaging revealed a large mass in the left adrenal gland.

A 4-phase computed tomography with intravenous contrast demonstrated a $7.7 \times 6.2 \times 7.7$ $\mathrm{cm}$ soft tissue mass involving the left adrenal gland abutting the gastric fundus. (Figure 2) Additionally, magnetic resonance imaging was performed to further characterize the adrenal mass which revealed low T2 signaling and mild heterogeneous enhancement with no obvious drop out on in and out of phase imaging and absolute washout of $63 \%$. Her preoperative hormonal workup was unremarkable with aldosterone at $2.1 \mathrm{ng} / \mathrm{dL}$ (ref: $0-30 \mathrm{mg} / \mathrm{dL}$ ). Urinary catecholamines were also within normal limits with epinephrine at $3 \mathrm{mcg}$ (ref: 0-20 mcg), norepinephrine at $42 \mathrm{mcg}$ ref: $0-135 \mathrm{mcg}$ ), dopamine at $310 \mathrm{mcg}$ (ref: $0-510 \mathrm{mcg}$ ).

A laparoscopic left adrenalectomy was performed with final pathology demonstrating a $9 \times 7 \times 5.5 \mathrm{~cm}$ non-small cell neoplasm with mitotic activity and necrosis consistent with high-grade adrenocortical carcinoma. Margins were adequate and negative. The pathologic stage was Stage II (T2, NO, MO).

Post operatively, the patient has undergone surveillance with CT imaging every 3 months for 1 year after surgery. Additionally, she was referred to an experienced quaternary center that corroborated the surveillance plan. The patient is currently 4 years post-operative surgical resection disease free.

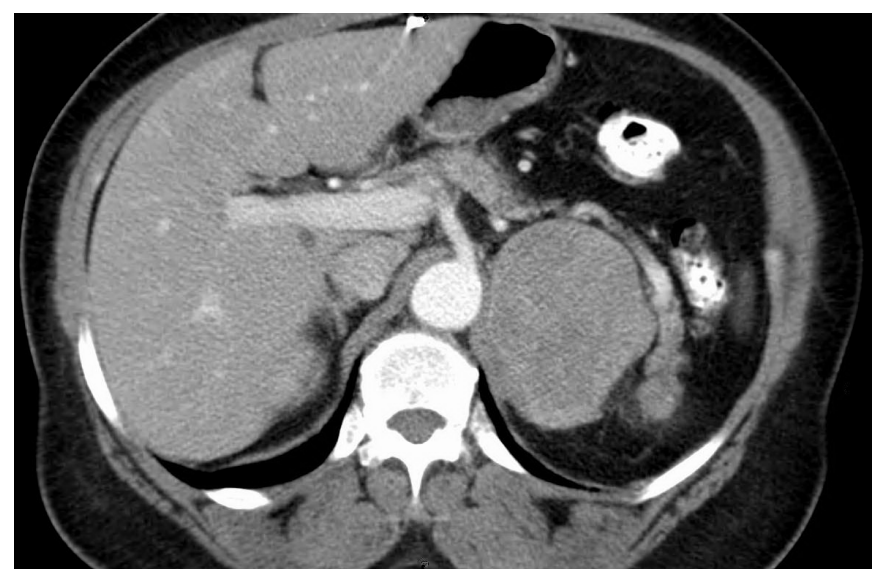

Figure 2. CT abdomen/pelvis revealed a left adrenal mass. 


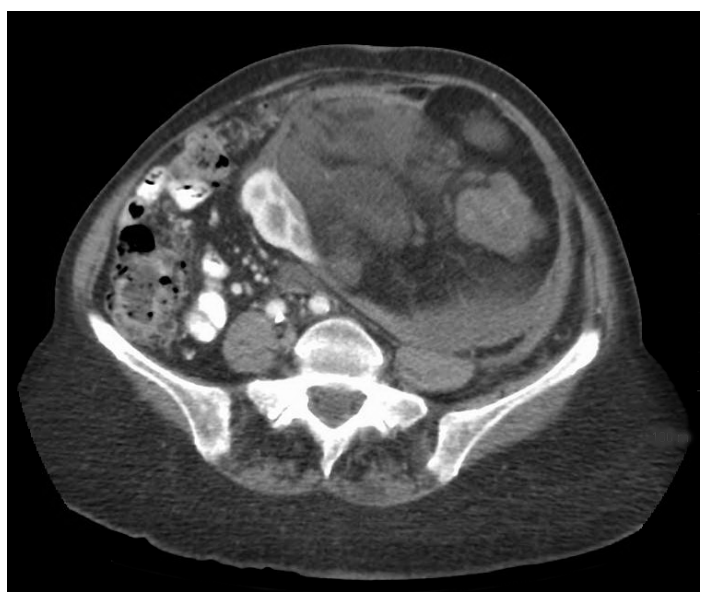

Figure 3. CT abdomen/pelvis revealed a left adrenal mass.

\section{Case 3}

An 87-year-old female presented with symptoms of abdominal discomfort, weight loss and an abdominal mass that was discovered on self-examination. Past medical history was significant for atrial fibrillation and breast cancer. Family history included a son and granddaughter with melanoma. There was no history of trauma, tobacco or alcohol use and no symptoms of functioning adrenal tumor.

Computed tomography (Figure 3) was performed revealing a mixed density partially enhancing mass within the left upper quadrant adjacent to the kidney with cystic and solid components with calcifications measuring 17.6 $\times 11.1 \times 26.0 \mathrm{~cm}$. The mass displaces the spleen anteriorly and the left kidney, aorta and bowel are displaced to the right hemiabdomen.

Given the patient's age and comorbidities, image guided core needle biopsy was offered. However, secondary to ongoing pain the decision was made with the patient to proceed with surgical resection. Preoperative biochemical evaluation was within normal limits.

After standard preoperative assessment and medical optimization, a radical resection of the retroperitoneal mass was performed. This included a distal pancreatectomy, splenectomy, left nephrectomy and left adrenalectomy, which were performed to resect the mass en bloc. Pathology demonstrated a grade 3 dedifferentiated liposarcoma with $\mathrm{R} 1$ resection margins. The pathologic stage was Stage III (T4, N1, MO). Patient received adjuvant radiation therapy due to the high grade of the sarcoma. Post operative surveillance imaging at 18 months revealed a $4 \mathrm{~cm}$ lesion in her resection bed which also had PET avidity suggesting recurrence. She subsequently underwent an endoscopic ultrasound guided FNA of the mass that confirmed recurrence.

\section{Case 4}

A 41-year-old female with multiple symptoms, including abdominal pain, flushing, sweating and weight loss, presented to her primary care physician. Abdominal examination was unremarkable. An abdominal ultrasound was performed revealing a $7 \times 6 \times 7 \mathrm{~cm}$, solid, echogenic mass between the liver and right kidney. This prompted a CT abdomen/pelvis with IV contrast (Figure 4), which demonstrated a $6 x$ $7 \mathrm{~cm}$ lesion of the right adrenal gland with mild internal soft tissue density.

A right adrenal resection was performed laparoscopically with removal of a necrotic and hemorrhagic tumor. Pathology showed a $6 \mathrm{~cm}$ mass with mildly elevated increase in dopamine/epinephrine. Pathology demonstrated a myelolipoma.

Adrenal myelolipoma is a rare benign tumor typically with lipid tissue containing a bone marrow component. Expanded use of imaging is leading to increased detection of myelolipomas. Generally, they are considered non-func- 


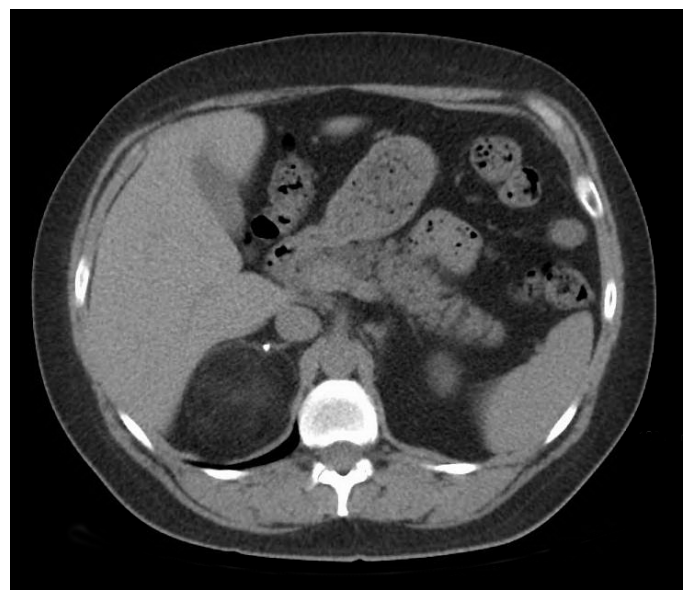

Figure 4. CT abdomen/pelvis revealed a left adrenal mass.

tional and do not require surgical excision. Additionally, they can be regarded as an exception to the mandatory metabolic workup for a newly detected adrenal mass, ${ }^{18,19}$ but rarely they can be functional. Given the patient's presenting symptoms, laparoscopic surgical excision was warranted, in accordance with the accepted indications for surgical excision of a myelolipoma, including symptomatic tumor $>4$ $\mathrm{cm}$, metabolically active tumor and suspicion of malignancy on imaging. ${ }^{20}$

\section{Case 5}

A 79-year-old female presented to the emergency department with shortness of breath and chest pain. Initially, she was treated for presumed acute congestive heart failure and pulmonary edema. However, the patient did not improve despite appropriate therapy.

This prompted CT imaging of the chest which revealed a partially visualized incidentaloma superior to the left kidney. An MRI of the abdo- men (Figure 5) revealed a partially calcified 9.5 $\times 8.6 \times 9.7 \mathrm{~cm}$ heterogeneously and peripherally enhancing mass arising from the left adrenal gland with central necrosis. There was no history of trauma. Laboratory studies demonstrated the tumor to be functional with elevated serum dopamine at $33 \mathrm{pg} / \mathrm{mL}$ (ref: 0-20 pg/mL) and norepinephrine at $1051 \mathrm{pg} / \mathrm{mL}$ (ref: $80-520$ $\mathrm{pg} / \mathrm{mL}$ ). Of note, epinephrine was normal at $17 \mathrm{pg} / \mathrm{mL}$ (ref: $10-100 \mathrm{pg} / \mathrm{mL}$ ). Urinary metanephrine was normal at $126 \mathrm{ug} / \mathrm{dL}$ (ref: 39-143 $\mathrm{ug} / \mathrm{dL}$ ), though urinary normetanephrine was elevated at $1548 \mathrm{ug} / \mathrm{dL}$ (ref: 109-393 ug/dL).

Given the heterogeneous tumor appearance on MRI with peripherally enhancing central necrosis, adrenal carcinoma could not be ruled out, so resection was recommended. Caution was taken due to the possibility of pheochromocytoma and alpha-blockade with phenoxybenzamine was administered. The patient was then taken to the operating room for a

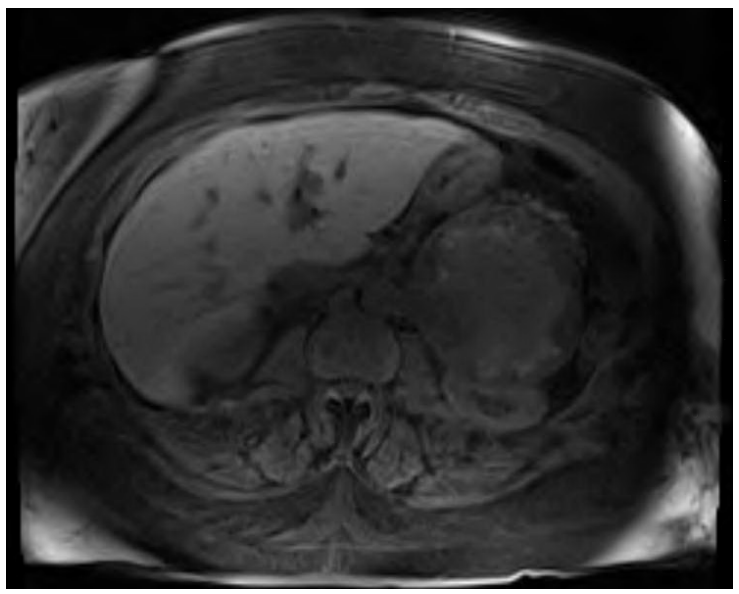

Figure 5. CT abdomen/pelvis revealed a left adrenal mass. 
left radical adrenalectomy. Given the tumor's encroachment on the left renal artery, the diminutive size of the left kidney, the patient's age and comorbidities, and a functioning right kidney; a left nephrectomy was also performed. The lesion measured $14 \times 13 \times 14 \mathrm{~cm}$. Pathology indicated the mass to be a benign hemorrhagic adrenal cyst with focal myelolipomatous metaplasia. The patient tolerated the surgery well and was discharged home. Ultimately, she died 40 days later due to complications of pre-existing COPD and chronic kidney disease.

\section{Discussion}

The diagnosis of adrenocortical carcinoma is difficult to make based upon pre-operative imaging alone. These cases demonstrate the importance of a comprehensive history and physical exam in determining the nature of a mass. Pertinent positives, including hypertension, headaches, hirsutism, etc., on review of symptoms, may reveal a functional tumor. When an adrenal mass is seen it is imperative to characterize the mass with cross-sectional imaging and obtain labs.

In patients with adrenocortical carcinoma, CT is more likely to reveal a tumor greater than $6 \mathrm{~cm}$ in diameter, reduced contrast washout on contrast CT, calcifications, heterogeneity, central low attenuation and poorly-defined margins..$^{12}$ A contrast CT showing greater than 10 Hounsfield Units (HU), as mentioned earlier, has been considered a predictor for malignancy, although some have suggested this threshold be changed to $13 \mathrm{HU} .{ }^{11}$

Lab work can aid in preoperative diagnosis and includes a full endocrine workup, including studies to rule out hypercortisolism, adrenocorticotropic hormone measurement and aldosterone-renin ratio. Additional tests to consider include dehydroepiandrosterone sulfate level, which is often elevated in malignant adrenal tumors, and plasma metanephrine and normetanephrine measurement, which can rule out pheochromocytoma. ${ }^{21}$

Despite the recommendations in place for diagnosing ACC based on imaging and labs, the diagnosis is frequently missed preoperatively, and improvement is needed in this field. ${ }^{22}$ One area in which further research must be done is in patient selection for preoperative biopsy, since imaging characterization has become quite sensitive and specific.11, 17 When an adrenal cancer is suspected, FNA biopsy is relatively contraindicated due to the risk of seeding the tumor. ${ }^{16}$ Therefore, when malignancy is a differential diagnosis, resection without biopsy is often the preferred treatment.

Several new modalities have been suggested for improving the capability to diagnose ACC preoperatively. New imaging methods, such as FDG-PET scan or PET using C-labeled metomidate (MTO), have shown some utility in diagnosing cortical tumors. ${ }^{23}, 24$ FDG-PET has been shown to diagnose ACCs with very high sensitivity and specificity, and therefore, is becoming more widely used. However, the efficacy of its routine use is unclear. ${ }^{23,24}$ Several molecular markers for ACC are currently being explored. MiRNAs 483-5p and 483-3p are generally overexpressed in ACC, and overexpression of miR483, miR-503 and miR-1275 are associated with decreased survival. ${ }^{25,26}$ Detection of aberrant methylation patterns and abnormal gene expression may also provide new methods of diagnosing adrenocortical carcinoma. ${ }^{4,26}$ As these methods become validated and more widely available for clinical use, clinicians may be able to diagnose ACC more efficiently, improving survival length and cure rates for patients with the disease.

The surgical goal in treatment of ACC is complete resection with negative margins, which offers the best and possibly the only chance at survival. In fact, a study of 3,982 patients by Bilimoria et al. showed that patients with margin-negative resections have a 44.2-month greater median survival than patients who undergo margin-positive resections. ${ }^{27}$ Although laparoscopic adrenalectomy (LA) is generally the best option for benign adrenal tumors, it has been shown to increase the likelihood of having positive margins compared to open adrenalectomy when used on patients with ACC, thus decreasing patients' chances for a cure. ${ }^{28}$ Studies have found lower overall survival in patients with stage II and greater ACC who undergo a laparoscopic approach, along with a higher risk of peritoneal carcinomatosis at the time of recurrence, which decreases the likelihood of a successful salvage surgery. ${ }^{28-31}$ 
Adrenal artery embolization is a minimally invasive procedure that can be used as an alternative or adjunct to surgery. Of the varied indications for this procedure, it is most often performed for oncologic palliation, such as pain relief, reduction of tumor bulk or preoperative reduction of tumor bulk. Emergency embolization for the hemostasis of ruptured tumors with retroperitoneal hemorrhage is an additional application. ${ }^{32}$ This procedure is not routinely used in the management of ACC but can be an adjunct with surgery in massive hemorrhagic tumors.

Due to the discouraging prognosis, Autorino et al. argues that open resection of ACC is the best approach, with laparoscopic resection being saved for a select few cases. ${ }^{33}$ On the other hand, Vanbrugghe et al. supports the use of laparoscopic adrenalectomy, especially for patients with smaller tumors and no evidence of invasion. This group argues that the extent of the resection is the important variable, and that if a complete resection with negative margins can be achieved using a laparoscopic approach, this is a viable option. ${ }^{34}$ Case 2 in our series could be regarded as support for this opinion. In that case, a laparoscopic approach was attempted despite imaging consistent with malignancy, and the intervention successfully achieved negative margins. The patient has shown no sign of recurrence since 2014. Furthermore, the exclusion of a laparoscopic approach as an option rests on the assumption that the ACC will be diagnosed and staged with invasive disease ruled out. Therefore, in cases that ultimately do not turn out to be ACC, the open approach is likely unnecessary. This again highlights the importance of improving methods of preoperative diagnosis.

In our series we describe five cases:

Case one was highly suspicious preoperatively for ACC and was approached aggressively. In most instances the kidney can be salvaged; however, complete lymphadenectomy is crucial and in this case, resulted in nephrectomy, due to the necessary resection of nodes around hilar vessels.

Case two was managed as a nonfunctional adrenal mass at $7 \mathrm{~cm}$ and thus, was approached laparoscopically with oncologic principles to resect en bloc or convert to open which was not required.

Case three represents an aggressive sarcoma. A preoperative biopsy with radiation may have been a suitable alternative, although the radiation field would have been quite large. It was believed on imaging to represent bleeding into a tumor, causing the pain and; therefore, a surgical option was advised.

Case four represented a symptomatic myelolipoma causing abdominal pain, sweating and weight loss. The tumor was excised via laparoscopic resection.

Case five demonstrated benign pathology with false elevation of normetanephrine. It is possible this false elevation occurred as a medication side effect. The patient was taking a beta- 2 agonist inhaler, which has been shown to cause false elevation of normetanephrines in the literature..$^{35}$ Regardless, these lesions can undergo angioembolization if classic imaging characteristics suggest angiomyolipoma. Transarterial embolization of angiomyolipoma demonstrates low rates of mortality and serious complications. ${ }^{36}$

\section{Conclusion}

In this case series we have highlighted the challenges and complexities of preoperatively diagnosing Adrenocortical Carcinoma and choosing the best operative approach. Further research is needed to improve preoperative diagnosis of ACC with imaging and laboratory assessment. This could potentially be accomplished through a randomized, prospective trial separating patients by pre-operative imaging modality and laboratory studies, but would require a well-organized, multi-center study given its overall low incidence in any given population.

\section{Conflicts of Interest}

The authors declare they have no conflicts of interest.

Drs. Jo Cooke-Barber, Andrew Schwemmer and Christopher Senkowski are employees of Memorial Health University Medical Center, a hospital affiliated with the journal's publisher.

This research was supported (in whole or in 
part) by HCA Healthcare and/or an HCA Healthcare affiliated entity. The views expressed in this publication represent those of the author(s) and do not necessarily represent the official views of HCA Healthcare or any of its affiliated entities.

\section{Author Affiliations}

1. Memorial Health University Medical Center

2. Ohio State University School of Medicine

3. Mercer University School of Medicine

\section{References}

1. Clark OH, Duh Q-Yang. Textbook of Endocrine Surgery. Philadelphia: W.B. Saunders; 1997.

2. Allolio B, Fassnacht M. Adrenocortical carcinoma: Clinical update. J Clin Endocrinol Metab. 2006;91(6):2027-2037. https://doi.org/10.1210/ jc. 2005-2639

3. Libé R. Adrenocortical carcinoma (ACC): diagnosis, prognosis, and treatment. Front Cell Dev Biol. 2015;3:45. https://doi.org/10.3389/ fcell.2015.00045

4. Szyszka P, Grossman AB, Diaz-Cano S, Sworczak K, Dworakowska D. Molecular pathways of human adrenocortical carcinomatranslating cell signalling knowledge into diagnostic and treatment options. Endokrynol Pol. 2016;67(4):427-450. https://doi.org/10.5603/ EP.a2016.0054

5. Ribeiro RC, Sandrini F, Figueiredo B, et al. An inherited p53 mutation that contributes in a tissue-specific manner to pediatric adrenal cortical carcinoma. Proc Natl Acad Sci U S A. 2001;98(16):9330-9335. https://doi.org/10.1073/ pnas. 161479898

6. Jouinot A, Bertherat J. MANAGEMENT OF ENDOCRINE DISEASE: Adrenocortical carcinoma: differentiating the good from the poor prognosis tumors. Eur J Endocrinol. 2018;178(5):R215-R230. https://doi.org/10.1530/ EJE-18-0027

7. Ayala-Ramirez M, Jasim S, Feng L, et al. Adrenocortical carcinoma: clinical outcomes and prognosis of 330 patients at a tertiary care center. Eur J Endocrinol. 2013;169(6):891-899. https://doi.org/10.1530/EJE-13-0519

8. Li L, Yang G, Zhao L, et al. Baseline Demographic and Clinical Characteristics of $\mathrm{Pa}-$ tients with Adrenal Incidentaloma from a Single Center in China: A Survey. Int J Endocrinol. 2017;2017:3093290. https://doi. org/10.1155/2017/3093290

9. Creemers SG, Hofland LJ, Korpershoek E, et al. Future directions in the diagnosis and medical treatment of adrenocortical carcinoma. Endocr Relat Cancer. 2016;23(1):R43-R69. https://doi.
org/10.1530/ERC-15-0452

10. Sturgeon C, Shen WT, Clark OH, Duh QY, Kebebew E. Risk assessment in 457 adrenal cortical carcinomas: how much does tumor size predict the likelihood of malignancy? J Am Coll Surg. 2006;202(3):423-430. https://doi.org/10.1016/j. jamcollsurg.2005.11.005

11. Ilias I, Sahdev A, Reznek RH, Grossman AB, Pacak K. The optimal imaging of adrenal tumours: a comparison of different methods. Endocr Relat Cancer. 2007;14(3):587-599. https://doi. org/10.1677/ERC-07-0045

12. Zhang HM, Perrier ND, Grubbs EG, et al. CT features and quantification of the characteristics of adrenocortical carcinomas on unenhanced and contrast-enhanced studies. Clin Radiol. 2012;67(1):38-46. https://doi.org/10.1016/j. crad.2011.03.023

13. Young WF Jr. Conventional imaging in adrenocortical carcinoma: update and perspectives. Horm Cancer. 2011;2(6):341-347. https://doi. org/10.1007/s12672-011-0089-z

14. lerardi AM, Petrillo M, Patella F, et al. Interventional radiology of the adrenal glands: current status. Gland Surg. 2018;7(2):147-165. https:// doi.org/10.21037/gs.2018.01.04

15. Elsayes KM, Mukundan G, Narra VR, et al. Adrenal masses: mr imaging features with pathologic correlation. Radiographics. 2004;24 Suppl 1:S73-S86. https://doi.org/10.1148/rg.24si045514

16. Berruti A, Baudin E, Gelderblom H, et al. Adrenal cancer: ESMO Clinical Practice Guidelines for diagnosis, treatment and follow-up. Ann Oncol. 2012;23 Suppl 7:vii131-vii138. https://doi. org/10.1093/annonc/mds231

17. Terzolo M, Stigliano A, Chiodini I, et al. AME position statement on adrenal incidentaloma. Eur J Endocrinol. 2011;164(6):851-870. https:// doi.org/10.1530/EJE-10-1147

18. NIH state-of-the-science statement on management of the clinically inapparent adrenal mass ("incidentaloma"). NIH Consens State Sci Statements. 2002;19(2):1-25.

19. Zeiger MA, Thompson GB, Duh QY, et al. American Association of Clinical Endocrinologists and American Association of Endocrine Surgeons Medical Guidelines for the Management of Adrenal Incidentalomas: executive summary of recommendations. Endocr Pract. 2009;15(5):450-453. https://doi.org/10.4158/ EP.15.5.450

20. Shenoy VG, Thota A, Shankar R, Desai MG. Adrenal myelolipoma: Controversies in its management. Indian J Urol. 2015;31(2):94-101. https://doi.org/10.4103/0970-1591.152807

21. Héron E, Chatellier G, Billaud E, Foos E, Plouin PF. The urinary metanephrine-to-creatinine ratio for the diagnosis of pheochromocytoma. Ann Intern Med. 1996;125(4):300-303. https:// doi.org/10.7326/0003-4819-125-4-199608150- 
$\underline{00008}$

22. Ozsari L, Kutahyaliouglu M, Elsayes EM, et al. Preexisting adrenal masses in patients with adrenocortical carcinoma: clinical and radiological factors contributing to delayed diagnosis. Endocrine. 2016;51(2):351-359. https://doi. org/10.1007/s12020-015-0694-7

23. Boland GW, Dwamena BA, Jagtiani Sangwaiya $M$, et al. Characterization of adrenal masses by using FDG PET: a systematic review and meta-analysis of diagnostic test performance. Radiology. 2011;259(1):117-126. https://doi. org/10.1148/radiol.11100569

24. Takeuchi S, Balachandran A, Habra MA, et al. Impact of ${ }^{18} \mathrm{~F}$-FDG PET/CT on the management of adrenocortical carcinoma: analysis of 106 patients. Eur J Nucl Med Mol Imaging. 2014;41(11):2066-2073. https://doi.org/10.1007/ s00259-014-2834-3

25. Soon PS, Tacon LJ, Gill AJ, et al. miR-195 and miR-483-5p Identified as Predictors of Poor Prognosis in Adrenocortical Cancer. Clin Cancer Res. 2009;15(24):7684-7692. https://doi. org/10.1158/1078-0432.CCR-09-1587

26. Özata DM, Caramuta S, Velázquez-Fernández $D$, et al. The role of microRNA deregulation in the pathogenesis of adrenocortical carcinoma. Endocr Relat Cancer. 2011;18(6):643-655. Published 2011 Oct 27. https://doi.org/10.1530/ ERC-11-0082

27. Bilimoria KY, Shen WT, Elaraj D, et al. Adrenocortical carcinoma in the United States: treatment utilization and prognostic factors. Cancer. 2008;113(11):3130-3136. https://doi.org/10.1002/ cncr.23886

28. Huynh KT, Lee DY, Lau BJ, Flaherty DC, Lee J, Goldfarb M. Impact of Laparoscopic Adrenalectomy on Overall Survival in Patients with Nonmetastatic Adrenocortical Carcinoma. J Am Coll Surg. 2016;223(3):485-492. https://doi. org/10.1016/j.jamcollsurg.2016.05.015

29. Payabyab EC, Balasubramaniam S, Edgerly $M$, et al. Adrenocortical Cancer: A Molecularly Complex Disease Where Surgery Matters. Clin Cancer Res. 2016;22(20):4989-5000. https://doi. org/10.1158/1078-0432.CCR-16-1570

30. Leboulleux S, Deandreis D, Al Ghuzlan A, et al. Adrenocortical carcinoma: is the surgical approach a risk factor of peritoneal carcinomatosis?. Eur J Endocrinol. 2010;162(6):1147-1153. https://doi.org/10.1530/EJE-09-1096

31. Gonzalez RJ, Shapiro S, Sarlis N, et al. Laparoscopic resection of adrenal cortical carcinoma: a cautionary note. Surgery. 2005;138(6):1078-1086. https://doi.org/10.1016/i.surg.2005.09.012

32. Fowler AM, Burda JF, Kim SK. Adrenal artery embolization: anatomy, indications, and technical considerations. AJR Am J Roentgenol. 2013;201(1):190-201. https://doi.org/10.2214/ AJR.12.9507

33. Autorino R, Bove P, De Sio M, et al. Open Versus Laparoscopic Adrenalectomy for Adreno- and Oncological Outcomes. Ann Surg Oncol. 2016;23(4):1195-1202. https://doi.org/10.1245/ s10434-015-4900-x

34. Vanbrugghe C, Lowery AJ, Golffier C, Taieb D, Sebag F. Adrenocortical carcinoma surgery-surgical extent and approach. Langenbecks Arch Surg. 2016;401(7):991-997. https://doi. org/10.1007/s00423-016-1462-8

35. Pacak K, Del Rivero J. Pheochromocytoma imaging. In: Feingold KR, Anawalt B, Boyce A, et al., ed., Endotext [Internet]. South Dartmouth, MA: MDText.com, Inc.; 2013. https://doi. org/10.1530/endoabs.32.S14.2

36. Murray TE, Doyle F, Lee M. Transarterial Embolization of Angiomyolipoma: A Systematic Review. J Urol. 2015;194(3):635-639. https://doi. org/10.1016/j.juro.2015.04.081 